\title{
Tcf4 Is Involved in Subset Specification of Mesodiencephalic Dopaminergic Neurons
}

\author{
Simone Mesman *, Iris Wever and Marten P. Smidt*(D) \\ Swammerdam Institute for Life Sciences, FNWI, University of Amsterdam, 1098 XH Amsterdam, \\ The Netherlands; iris.wever@nijgeerten.nl \\ * Correspondence: s.mesman@uva.nl (S.M.); m.p.smidt@uva.nl (M.P.S.)
}

Citation: Mesman, S.; Wever, I.; Smidt, M.P. Tcf4 Is Involved in Subset Specification of Mesodiencephalic Dopaminergic Neurons. Biomedicines 2021, 9, 317. https://doi.org/ 10.3390/biomedicines 9030317

Academic Editor: Marc Ekker

Received: 14 December 2020

Accepted: 17 March 2021

Published: 20 March 2021

Publisher's Note: MDPI stays neutral with regard to jurisdictional claims in published maps and institutional affiliations.

Copyright: (c) 2021 by the authors. Licensee MDPI, Basel, Switzerland. This article is an open access article distributed under the terms and conditions of the Creative Commons Attribution (CC BY) license (https:// creativecommons.org/licenses/by/ $4.0 /)$.

\begin{abstract}
During development, mesodiencephalic dopaminergic (mdDA) neurons form into different molecular subsets. Knowledge of which factors contribute to the specification of these subsets is currently insufficient. In this study, we examined the role of Tcf4, a member of the E-box protein family, in mdDA neuronal development and subset specification. We show that Tcf4 is expressed throughout development, but is no longer detected in adult midbrain. Deletion of Tcf4 results in an initial increase in TH-expressing neurons at E11.5, but this normalizes at later embryonic stages. However, the caudal subset marker Nxph3 and rostral subset marker Ahd2 are affected at E14.5, indicating that Tcf4 is involved in correct differentiation of mdDA neuronal subsets. At P0, expression of these markers partially recovers, whereas expression of Th transcript and TH protein appears to be affected in lateral parts of the mdDA neuronal population. The initial increase in TH-expressing cells and delay in subset specification could be due to the increase in expression of the bHLH factor Ascl1, known for its role in mdDA neuronal differentiation, upon loss of Tcf4. Taken together, our data identified a minor role for Tcf4 in mdDA neuronal development and subset specification.
\end{abstract}

Keywords: midbrain development; dopamine neurons; Tcf4; subset specification; bHLH factors

\section{Introduction}

Mesodiencephalic dopaminergic (mdDA) neurons are thought to arise from neural progenitors in the ventricular zone (VZ) of the floor plate (FP) and basal plate (BP) of the midbrain from E11.5 onward [1-3]. When neural progenitors acquire an mdDA neuronal cell fate, they migrate toward their final location and gain a subset-specific identity [4-8]. Of these subsets, the neuroanatomically distinct substantia nigra (SNc) and ventral tegmental area (VTA) are best known, mainly due to the selective degeneration of the SNc during Parkinson's disease (PD) [9]. The specification of the different subsets in the mdDA system is dependent on a unique set of transcription factors and signaling cascades during development [4-8,10], although knowledge of the factors that are important during subset specification is currently insufficient.

One protein family important in neuronal differentiation is the basic helix-loop-helix (bHLH) family of transcription factors, an enormous protein family that can be subdivided into different subfamilies [11]. The function of bHLH proteins differs throughout embryonic development and is dependent on their spatial-temporal expression pattern and binding partner(s) $[12,13]$. Several members of the bHLH protein family, e.g., Ascl1 (Mash1), Ngn2, Tcf12, and Nato3, have been shown to be involved in early stages of mdDA neuronal development during fate decision of neural progenitors and later subset specification [14-17].

The E-box protein family is a small subfamily of the bHLH protein family, and contains 3 family members; Tcf3 (E2A), Tcf4 (E2-2), and Tcf12 (Heb) [18], which are expressed throughout the developing embryo and embryonic brain. These proteins are known to specifically bind to a so-called E-box (CANNTG) in the promoter of different genes, and can act as an activating or repressive factor via homo- and heterodimerization with other 
bHLH factors [12,13]. Most studies of the function of E-box proteins focus on their role in immune-system development. They have been found to be critical for the differentiation from $\mathrm{CD} 4^{+} \mathrm{CD}^{+}$double-positive $\mathrm{T}$ cells to either $\mathrm{CD} 4^{+}$or $\mathrm{CD} 8^{+}$single-positive $\mathrm{T}$ cells [19]. However, each E-box protein plays a unique role in immune-system development, and loss of Tcf4 results in a milder phenotype than loss of Tcf12 or Tcf3 $[19,20]$.

During brain development, E-box proteins are thought to regulate common targets for neurogenesis and unique target genes for neuronal subtype development, dependent on their interaction partner and expression area [13]. Fischer et al. [21] have shown that E-box proteins may function together with Ascl1 to fine-tune the differentiation progression in the subventricular zone of the adult forebrain during postnatal neurogenesis. Furthermore, loss of Tcf12 and Tcf 3 leads to an overall reduction in brain size, although the morphology of brain structures is not altered [22], and loss of Tcf12 during midbrain development has been shown to result in a delay in mdDA neuronal differentiation and ultimately affected subset development [16]. Tcf4 has been implicated in several neurodevelopmental diseases, like autism and schizophrenia, and loss of one copy of Tcf4 leads to major brain malformations resulting in Pitt-Hopkins Syndrome in humans [23,24]. During murine brain development, Tcf 4 has been shown to have a pivotal role in development of the cortex, hippocampus, pontine nucleus, and major axonal tracts like the corpus callosum and anterior commissure [25-27].

In this study, we focus specifically on the role of Tcf4 in the development of the mdDA neuronal system. In the rat brain, TCF4 has been shown to interact with CDP2 (the rat homologue for $\mathrm{Cux}$ genes in mice) [28]. This interaction is thought to relieve the repression of CDP2 on the Th gene, thereby initiating Th transcription [28]. Contrary to what was detected in rat, we did not find a decrease in TH expression in the mice Tcf4 mutant midbrain during development. We detected a transient upward trend in the amount of TH-expressing cells at E11.5, which was no longer apparent at E12.5 and normalized at E14.5. However, expression of the subset specific markers Ahd2 (rostral subset) and Nxph3 (caudal subset) was strongly affected at this developmental stage, which partly recovered during terminal differentiation. It is possible that the early increase in the amount of $\mathrm{TH}^{+}$ cells in the midbrain and affected expression of subset markers is due to upregulation of the bHLH factor Ascl1 upon loss of Tcf4. Taken together, we have established the E-box protein Tcf4 as a novel factor involved in correct development of mdDA neurons.

\section{Experimental Section}

\subsection{Ethics Statement}

All animal studies were performed in accordance with local animal-welfare regulations, as this project was approved by the animal experimental committee (Dier ethische commissie, Universiteit van Amsterdam; DEC-UvA), and international guidelines.

\subsection{Animals}

The transgenic mouse line B6;129-Tcf 4 tm1Zhu /J originated from the Jackson Laboratory. B6;129-Tcf $4^{\text {tm1Zhu } / J ~ m o u s e ~ l i n e ~ w a s ~ b a c k-c r o s s e d ~ w i t h ~ t h e ~ C 57 B L / 6 ~ l i n e ~ a n d ~ W T ~ a n d ~}$ mutant littermate embryos to study the effects of the loss of Tcf4 on mdDA neuronal development, were generated by crossing heterozygous Tcf4 mice. To study the WT expression pattern of Tcf4, WT embryos were generated by crossing C57BL/6 mice. Pregnant mice (embryonic day 0.5 (E0.5) is defined as the morning of plug formation) were sacrificed by cervical dislocation. Embryos were collected in 1× PBS and immediately frozen on dry ice, or fixed by immersion of 3-12 h in $4 \%$ paraformaldehyde (PFA) at $4{ }^{\circ} \mathrm{C}$. After PFA incubation, samples were cryoprotected $\mathrm{O} / \mathrm{N}$ at $4{ }^{\circ} \mathrm{C}$ in $30 \%$ sucrose. Embryos were frozen on dry ice and stored at $-80^{\circ} \mathrm{C}$. Cryosections were sliced at $16 \mu \mathrm{m}$, mounted on Superfrost Plus slides (Thermo Fisher Scientific, Waltham Massachussetts, USA), air-dried, and stored at $-80{ }^{\circ} \mathrm{C}$ until further use. 


\subsection{Genotyping}

Genotyping of B6;129-Tcf $4^{\text {tm1Zhu }} / \mathrm{J}$ transgenic embryos and mice was performed according to the protocol of the Jackson Laboratory. Briefly, $100 \mathrm{ng}$ of genomic DNA was used together with primer pair: FP 5'-AGCGCGAGAAAGGAACGGAGGA-3', RP1 5'-GGCAATTCTCGGGAGGGTGCTT-3' ${ }^{\prime}$, and RP2 5'-CCAGAAAGCGAAGGAGCA-3', resulting in a product at $229 \mathrm{bp}$ for the $\mathrm{WT}$ allele and a product at $400 \mathrm{bp}$ for the $\mathrm{KO}$ allele.

\subsection{In Situ Hybridization and Combined TH-DAB IHC}

In situ hybridization with digoxigen (DIG)-labeled probes was performed as described previously [29]. Fresh frozen sections were fixed in 4\% PFA for $30 \mathrm{~min}$ and acetylated with $0.25 \%$ acetic anhydride in $0.1 \mathrm{M}$ triethanolamine for $10 \mathrm{~min}$. Probe hybridization was carried out at $68^{\circ} \mathrm{C} \mathrm{O} / \mathrm{N}$ with a probe concentration of $0.4 \mathrm{ng} / \mu \mathrm{L}$ in a hybridization solution containing $50 \%$ deionized formamide, $5 \times$ SSC, $5 \times$ Denhardt's solution, $250 \mu \mathrm{g} / \mathrm{mL}$ tRNA Baker's yeast, and $500 \mu \mathrm{g} / \mathrm{mL}$ sonificated salmon sperm DNA. The following day, slides were washed in $0.2 \times$ SSC for $2 \mathrm{~h}$ at $68{ }^{\circ} \mathrm{C}$ followed by blocking with $10 \%$ HIFCS in buffer $1(100 \mathrm{mM}$ Tris Hcl, pH 7.4 and $150 \mathrm{mM} \mathrm{NaCl})$ for $1 \mathrm{~h}$ at RT. DIG-labeled probes were detected by incubating with an alkaline-phosphatase-labeled anti-DIG antibody (Roche, Mannheim, Germany, 1:5000), using NBT-BCIP as a substrate. If in situ hybridization was not followed by TH-DAB immunohistochemistry, slides were washed $2 \times 5$ min in $\mathrm{T}_{10} \mathrm{E}_{5}$, dehydrated with ethanol, and embedded in Entellan.

DIG in situ hybridization was performed with the following probes: $918 \mathrm{bp}$ Tcf4 fragment bp 1101-2018 of mouse cDNA, 873 bp Ascl1 fragment bp 1452-2325 of mouse cDNA, 491 bp Th fragment bp 252-143 of mouse cDNA, 1792 bp Ahd2 fragment bp 5-1801 of mouse cDNA, and 480 bp Nxph3 fragment bp 1374-1854 of mouse cDNA.

After DIG in situ hybridization, Tcf4 stained WT sections were used for DAB immunohistochemistry on TH. Slides were incubated in $0.3 \% \mathrm{H}_{2} \mathrm{O}_{2}$ in Tris-buffered saline (TBS) for $30 \mathrm{~min}$ at RT. Thereafter, blocking was performed with $4 \%$ HIFCS in TBS. Slides were incubated $\mathrm{O} / \mathrm{N}$ with primary antibody Rb-TH (Pel-Freez, Rogers Arkansas, USA 1:1000) in TBS. The following day, slides were incubated for $1 \mathrm{~h}$ with goat-anti-rabbit biotinylated secondary antibody (Vector Laboratories, Burlingame, California, USA 1:1000) in TBS, followed by incubation with avidine-biotin-peroxidase reagents (ABC elite kit, Vector Laboratories, Burlingame, CA, USA 1:1000) in TBS. Slides were stained with DAB (3,3'-diamino-benzidine) for a maximum of $10 \mathrm{~min}$. Slides were dehydrated with ethanol and embedded with Entellan.

\subsection{Immunohistochemistry}

Fluorescence immunohistochemistry (IHC) was carried out as described previously $[30,31]$. Cryosections were blocked with either $4 \%$ HIFCS or $5 \%$ normal donkey serum (for sheep primary antibodies) in $1 \times$ THZT and incubated with a primary antibody $(\mathrm{Rb}-$ TH (Pel-Freez, Rogers Arkansas, USA 1:1000), Sh-TH (Millipore, Waltham, MA, USA AB1542, 1:1000), Rb-AHD2 (Abcam, Cambridge, UK, AB24343, 1:200), Rb-PITX3 ([32] 1:1000)), diluted in $1 \times$ THZT O/N at RT. The next day, sections were incubated with a secondary Alexafluor antibody (anti-rabbit, anti-sheep) diluted 1:1000 in 1× TBS for $2 \mathrm{~h}$ at RT. After immunostaining, nuclei were stained with DAPI (1:3000) and washed extensively in $1 \times$ PBS. Slides were embedded in Fluorsave (Calbiochem, Amsterdam, The Netherlands) and analyzed with the use of a fluorescent microscope (DM5500B Leica, Amsterdam, The Netherlands). The antibody against AHD2 required antigen retrieval as follows. Slides were incubated with $0.1 \mathrm{M}$ citrate buffer $\mathrm{pH} 6$ for $3 \mathrm{~min}$ at $800 \mathrm{~W}$ and $9 \mathrm{~min}$ a $400 \mathrm{~W}$, then cooled down to RT in a water bath, after which the protocol was followed as described above.

Quantification of TH- and AHD2-expressing cells at embryonic stages was performed as follows. TH- and AHD2-expressing cells were counted in 5-11 (matching) sagittal sections $\left(\mathrm{n}=3 \mathrm{WT} ; \mathrm{n}=3 \mathrm{KO}\right.$ ). Cells were counted as $\mathrm{TH}^{+}$or $\mathrm{AHD}^{+}$when staining co- 
localized with a nuclear DAPI staining. Statistical analysis was performed via a one- or two-tailed Student's $t$-test.

\subsection{Quantitative PCR ( $q P C R)$}

RNA was isolated from dissected E14.5 midbrain of Tcf4 WT and Tcf4 mutant embryos (littermates; at least two litters were used per experiment). Relative RNA expression levels were determined by qPCR real-time PCR (Lightcycler 480, Roche, Bazel, Switzerland) using the Quantitect SYBR Green PCR Lightcycler Kit (Qiagen, Hilden, Germany) according to the manufacturer's protocol. For each reaction, $10 \mathrm{ng}$ (dissected material) total RNA was used as input. Primer sequences are listed in Table S1.

Quantified qPCR results represent average values of experiments performed on $4 \mathrm{bi}$ ological samples for each genotype. The qPCR experiments required multiple litters to obtain a sufficient sample size. Measurements that differed by $\geq 2 \times$ standard deviation from the mean were excluded. Statistical analysis was performed via a two-tailed Student's t-test.

\section{Results}

\subsection{Tcf4 Is Expressed from E11.5 Onward and Partly Overlaps with the} TH-Expressing Population

Since bHLH factors are known to be important in mdDA neuronal development and the E-box protein family is expressed throughout the embryonic brain, we investigated the expression pattern of the E-box factor Tcf4 (E2-2) during development in the brain. Tcf4 was found to be expressed throughout the hindbrain, midbrain, and prosomere 1-3 during development (Figure 1A; anatomic maps in Figure 1A,B were adapted from the Allen Brain Atlas (http:/ / atlas.brain-map.org, accessed on 3 January 2019). At E11.5, Tcf4 showed a very low level of expression in the midbrain area, and its expression pattern was restricted to the ventricular zone (VZ) of both the floor plate (FP) and basal plate (BP) (Figure 1B). This expression pattern continued at E12.5, and overlap was detected between the Tcf4 transcript and TH protein in the FP area of the midbrain (Figure 1B). At E14.5, the expression of Tcf 4 increased and became more restricted to the rostral area in both the lateral and medial sections (Figure 1A), although Tcf4 showed a strong expression in the medial areas of the midbrain (Figure 1B). Although Tcf4 is expressed in the midbrain during embryonic development, it can no longer be detected in the adult midbrain. Taken together, the expression profile of Tcf4 suggests that it may have a role during early development of the mdDA neuronal population, but is not likely to have a role in adult mdDA neurons, as its expression was not detected at this stage.

\subsection{Loss of Tcf4 Leads to an Upward Trend in TH-Expressing Neurons at E11.5 but Not at E14.5,} and Sporadic Loss of PITX3 at E14.5

Above we have shown that Tcf4 is expressed throughout the midbrain during development and is specifically expressed in the VZ at early embryonic stages. MdDA neurons are shown to originate in the VZ of the FP and FP-BP boundary from E10.5 onward, with neuronal birth peaking between E11 and E12 [1,2,33]. To determine whether Tcf4 has a role in the early cell-fate commitment of neural progenitors toward an mdDA neuronal fate, we performed immunohistochemistry on TH at E11.5 Tcf4 mutant embryos [34] (Figure 2A, left panel). In the Tcf4 mutant midbrain, we detected an upward trend in TH-expressing neurons compared to WT embryos (Figure 2A, right panel; WT $\mathrm{n}=3$, mutant $\mathrm{n}=5 ; p=0.06$, one-tailed), suggesting an accelerated neuronal differentiation at E11.5.

To determine whether this accelerated neuronal differentiation early during development is persistent in later stages, we examined the anatomical build-up of the mdDA neuronal population at E12.5 and E14.5. The composition of the mdDA neuronal population already appeared to be normalized at E12.5 (Figure S1), and TH expression in the midbrain of the Tcf4 mutant at E14.5 was not visibly altered compared to its WT littermates (Figure 2B). Quantification of the TH-expressing neuronal population confirms that the amount of $\mathrm{TH}^{+}$neurons was similar between the Tcf4 WT $(\mathrm{n}=3)$ and mutant $(\mathrm{n}=3)$ 
embryos at E14.5. Although the TH-expressing population showed a normal anatomical build-up, in some Tcf4 mutant embryos, we detected a clear loss of PITX3, a factor specifically expressed in postmitotic dopamine neurons, in the midbrain, although PITX3 expression was still present in the musculature of these embryos (Figure 2C). This effect on PITX3 was only observed in a subset of animals at E14.5 (out of 3 embryos, expression of PITX3 was completely lost in 1 and partially lost in 2 embryos compared to their WT littermates), and was not observed at E13.5 and E15.5 (Figure 2C; n = 3 for all stages). Taken together, these data indicate that loss of Tcf4 results in a possible accelerated mdDA neuronal differentiation and a not fully penetrating effect on terminal differentiation.

A

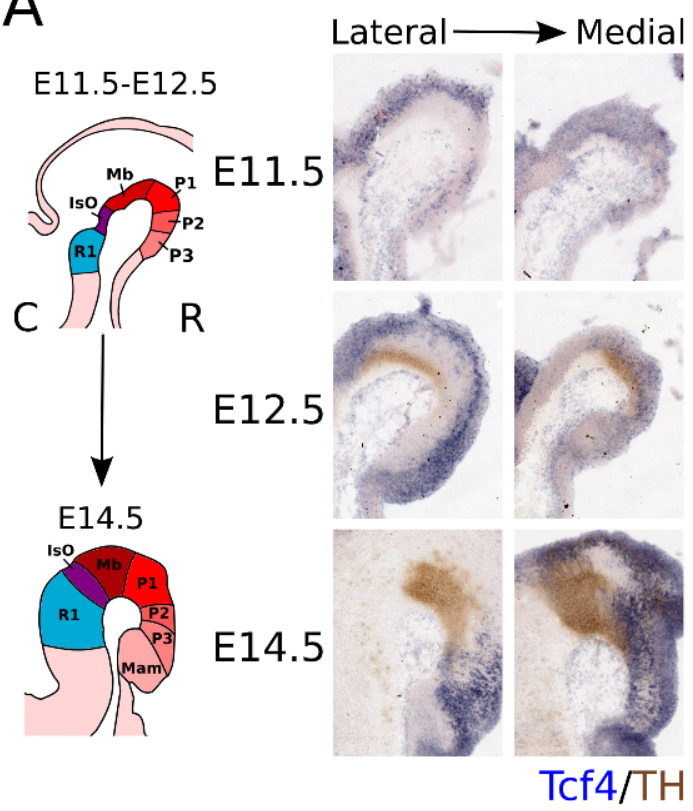

B

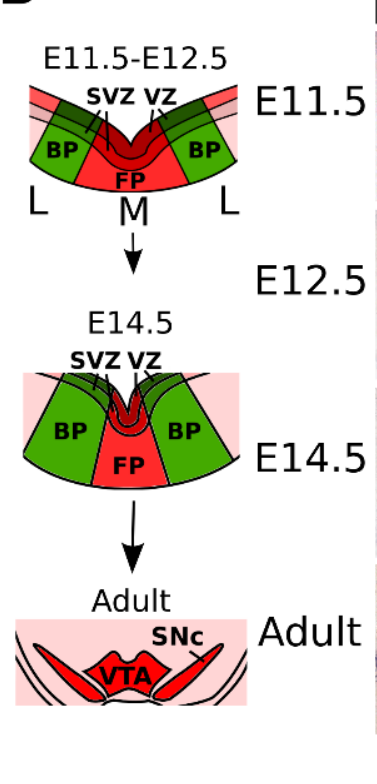

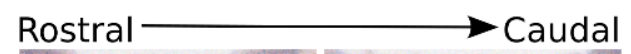

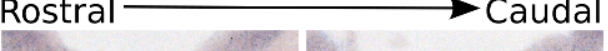

Figure 1. Tcf4 is expressed in the VZ of the developing midbrain and overlaps with part of the rostral TH-expressing population. (A) Sagittal view of Tcf4 expression throughout development. Tcf4 (blue) is expressed from E11.5 onward in the developing embryonic midbrain area. At E11.5, expression of Tcf 4. is specific for the VZ from R1-P3. At E12.5, its expression overlaps with the medial part of the TH-expressing neuronal population (brown), which is more apparent at E14.5 and even strongly overlaps with the rostral $\mathrm{TH}^{+}$population. Anatomic maps were adapted from the Allen Brain Atlas (http:/ / atlas.brain-map.org accessed on 3 January 2019). C: caudal, R: rostral. (B) Coronal view of Tcf4 expression throughout development and in the adult. Tcf4 (blue) shows expression in the VZ of the BP of the midbrain at E11.5. Expression in the FP is not clearly detected until E12.5. Overlap with the TH-expressing population (brown) can mainly be detected in medial parts of the midbrain at E12.5 and E14.5. In the adult midbrain, Tcf4 is no longer detected. Anatomic maps were adapted from the Allen Brain Atlas (http:/ /atlas.brain-map.org accessed on 3 January 2019). L: lateral, M: medial.

\subsection{Correct mdDA Neuronal Subset Specification Is Affected in the Tcf4 Mutant}

Above we have shown that loss of Tcf4 appears to have a possible effect on the start of mdDA neuronal differentiation, but does not clearly affect the TH-expressing population at later stages. However, expression of Tcf4 in specific areas of the midbrain overlapping with mdDA neurons and the possible effect on mdDA neuronal differentiation could point to a function in later differentiation and even result in a change in subset specification.

To determine whether Tcf4 may have a role in subset development of the mdDA neuronal population, we examined the expression levels of several rostral and caudal subset markers in E14.5 dissected midbrain material by means of qPCR (Figure 3A). Expression of the caudal markers Tle3 and Cck [8] was unaffected in the mdDA neuronal population, whereas the caudal marker Nxph3 [35] showed a decrease of $~ 50 \%$ in the Tcf4 mutant midbrain ( $\mathrm{n}=4 ; p=0.036$, two-tailed). Expression of the rostral markers Rspo2, Pbx3, Tle4, Ebf1, and Ebf2 were unaffected in the Tcf4 mutant midbrain $(n=4)[8,36]$. Expression of Rspo2 appeared to be downregulated with $\sim 25 \%$, but due to large variation between 
embryos, this was not significant ( $\mathrm{n}=4 ; p=0.35$, two-tailed). Ahd2 expression, on the other hand, showed a downregulation of $\sim 75 \%$ in the Tcf4 mutant ( $\mathrm{n}=4 ; p=0.0008$, two-tailed). The loss of Nxph3 in caudal areas and $A h d 2$ in rostral areas was confirmed via in situ hybridization at E14.5 (Figure 3B). Similar to Ahd2 transcript expression, AHD2 protein levels were strongly affected at E14.5 (Figure 3B).


Figure 2. The Tcf4 mutant shows an upward trend in the amount of $\mathrm{TH}^{+}$cells at E11.5, which normalizes at E14.5. (A) Loss of Tcf4 leads to an upward trend in TH-expressing cells (red) in the mutant embryo at E11.5. Quantification of the TH ${ }^{+}$ neurons shows an upward trend in Tcf4 mutant embryos (white bar) compared to WT embryos (black bar), although this is not significant (WT $n=3$; mutant $\mathrm{n}=5 ; p=0.06$ one-tailed). WT was set at $100 \%$. C: caudal, R: rostral. (B) Loss of Tcf4 has no apparent effect on the morphology and the presence of TH-expressing neurons (red) in the midbrain at E14.5. Quantification of $\mathrm{TH}^{+}$neurons in the WT (black bar) and Tcf4 mutant (white bar) midbrain does not show a difference in the amount of the TH-expressing neurons ( $n=3$; two-tailed). WT was set at $100 \%$. C: caudal, R: rostral. (C) Expression of PITX3 (green) is affected in some Tcf4 mutant embryos at different levels in the embryonic midbrain at E14.5. This effect on PITX3 was not seen in the musculature of the tested embryos. Furthermore, at E13.5 and E15.5 no clear difference in PITX3 (green) expression level or area was detected. C: caudal, R: rostral. 
A

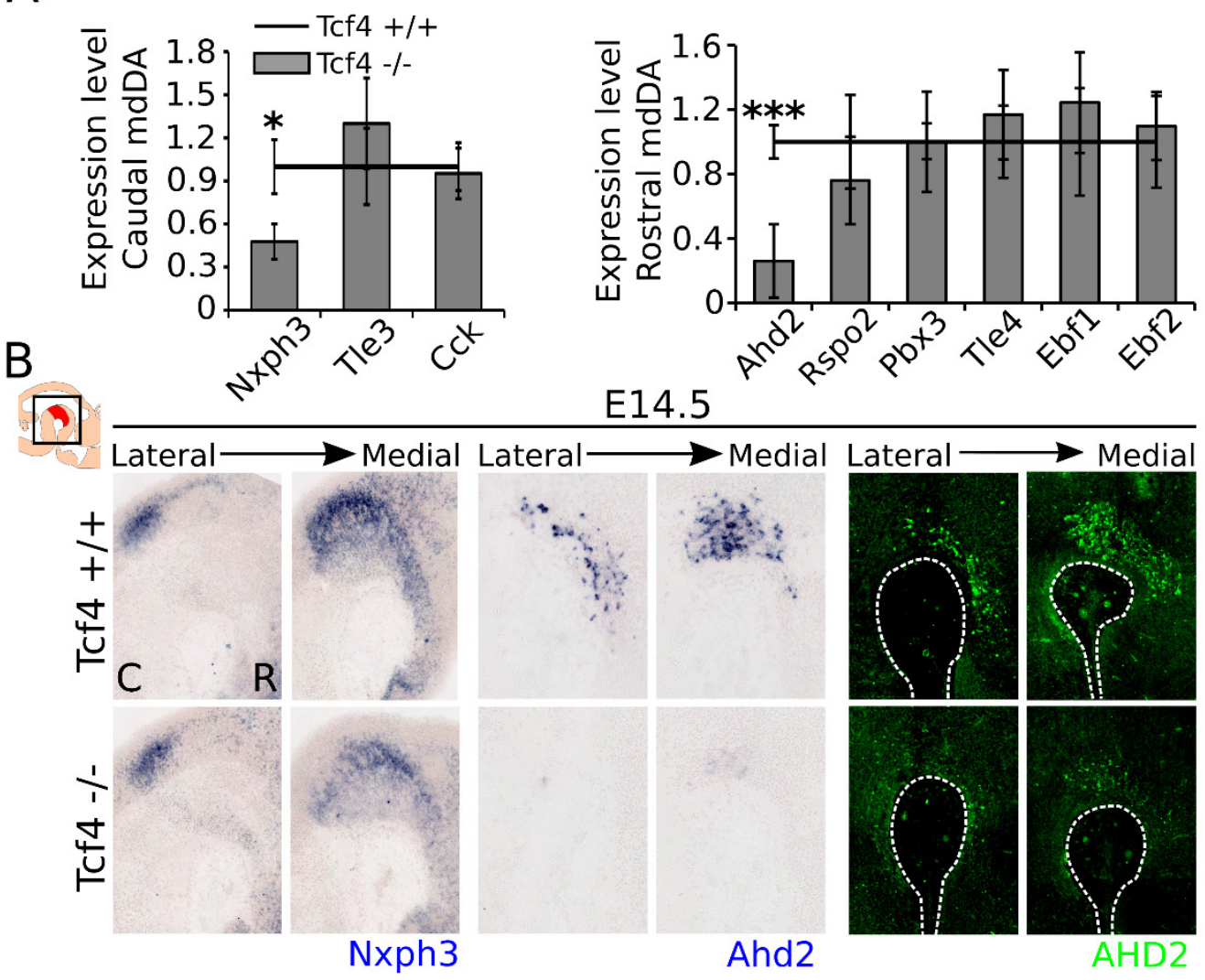

C



AHD2

E15.5
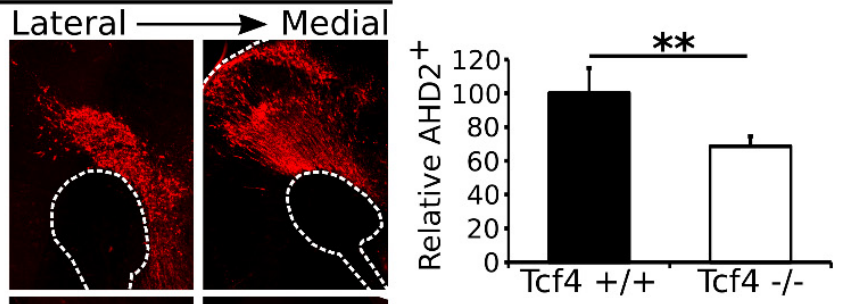
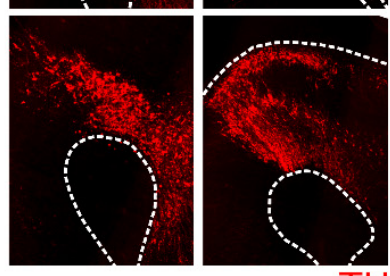

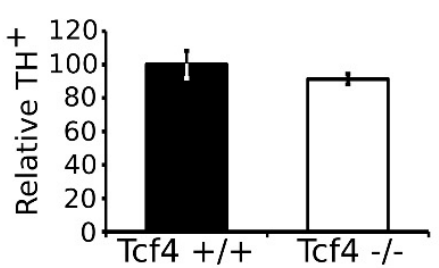

Figure 3. Expression of the caudal marker Nxph3 and the rostral marker Ahd2 is decreased in E14.5 Tcf4 mutant embryos, although expression of AHD2 partly recovers at E15.5. (A) qPCR of caudal and rostral markers in the mdDA system. Expression of caudal markers Tle3 and Cck is unaffected in the Tcf4 mutant ( $\mathrm{n}=4$; two-tailed). Expression of Nxph3, on the other hand, shows a significant decrease of $\sim 50 \%\left({ }^{*} \mathrm{n}=4 ; p=0.036\right.$ two-tailed). Expression of rostral markers Rspo2, $P b x 3, T l e 4, E b f 1$, and Ebf2 is not altered in the mutant ( $\mathrm{n}=4$; two-tailed). However, expression of the rostral marker Ahd2 is decreased with $\sim 75 \%$ in the mutant $\left({ }^{* * *} \mathrm{n}=4 ; p=0.0008\right.$ two-tailed). WT expression levels were set at 1 . (B) In situ hybridization of Nxph3 (blue, left panel) and Ahd2 (blue, right panel) confirms the qPCR data and shows that these markers are visibly altered in the midbrain of the Tcf4 mutant compared to WT littermates. AHD2 protein (green) expression is similarly affected in the Tcf4 mutant midbrain at E14.5. C: caudal, R: rostral. (C) At E15.5, the expression of AHD2 (green) is partly recovered. Quantification shows an $\sim 30 \%$ decrease in the amount of AHD2 ${ }^{+}$cells at E15.5 (** WT $n=3$, mutant $n=4$; $p=0.006$ two-tailed), although the expression of $\mathrm{TH}$ is not visibly altered and the amount of $\mathrm{TH}^{+}$cells is not significantly changed at this stage (black bars) (WT $n=3$, mutant $n=4$; one-tailed). WT was set at 100\%. C: caudal, R: rostral. 
At E15.5, some expression of AHD2 protein was detected, but loss of this protein was still detectable (Figure 3C). Quantification of AHD2-expressing neurons at E15.5 showed a decrease of $\sim 30 \%$ in the mutant (WT $n=3$, mutant $n=4 ; p=0.006$, one-tailed). Similar to what was detected at E14.5, the amount of TH-expressing neurons was not altered at E15.5 (WT $\mathrm{n}=3$, mutant $\mathrm{n}=4$ ), although the expression of subset-specific markers was clearly altered. These data showed that, although $\mathrm{TH}$-expressing neurons appear to be present in normal numbers at this stage in the Tcf4 mutant, the expression of specific rostral and caudal subset markers was affected upon loss of Tcf4.

\subsection{Ahd2 and Nxph3 Expression Partially Recovers Later in Development, Whereas Th Expression Becomes Slightly Affected at P0}

As shown above, loss of Tcf4 results in an initially changed subset specification as exemplified by the change in Ahd2 and Nxph3 levels. To examine whether the expression of these subset markers remains affected or recovers during mdDA development, we investigated the expression of Th, Ahd2, and Nxph3 at P0 (Figure 4). Although the expression of TH was not significantly altered at E14.5, the expression of Th at P0 appeared to be affected in the most lateral regions of the mdDA neuronal pool (Figure $4 \mathrm{~A}$, black arrowheads). Similar to the expression of Th transcript, the expression of TH protein appeared to be affected in the most lateral regions of the mdDA neuronal population (Figure 4A, white arrowheads). However, quantification of the TH-expressing cells in the SNc, VTA, and the total number of TH-expressing cells in the midbrain area shows that the amount of TH-expressing cells was not significantly altered (Figure 4A). Expression of Ahd2 was strongly decreased at E14.5, but recovered during embryonic development. At P0, the expression of Ahd2 was almost completely recovered, although some loss could still be detected in the most lateral regions of the mdDA neuronal population (Figure $4 \mathrm{~B}$, black arrowheads). Nxph3 showed a weak expression in the medial-caudal area of the mdDA neuronal system at P0, which was comparable to its expression at E14.5. However, similar to $A h d 2$, its expression appeared to be recovered in the mutant midbrain at $\mathrm{P} 0$, although in the most caudal sections expression of Nxph3 was still slightly affected (Figure $4 \mathrm{~B}$, black arrowheads). Pseudo-overlays of adjacent sections of the expression patterns of $T h$ and Ahd2, and of Th and Nxph3, showed that the expression of these markers overlapped in both WT and mutant brains, and that the loss of these markers was detected in similar areas (white arrowheads). Together, these data indicated that the expression of subset markers partially recovered during mdDA development. Even more so, expression of TH, which was not affected at E14.5, appeared to be decreased in the most lateral regions of the mdDA neuronal system at P0.

\subsection{Ascl1 Is Upregulated in the Developing Midbrain of the Tcf4 Mutant}

As shown above, loss of Tcf4 leads to an increase in the amount of TH-expressing neurons in the embryonic midbrain, which has no significant effect on the total TH-expressing population at E14.5, and a loss of expression of the subset markers Ahd2 and Nxph3, which partially recovers during development. It is known from other cell systems that loss of E-box proteins may be compensated by other E-box or bHLH proteins, and that these proteins are involved in the regulation of expression of each other $[13,15]$. To determine whether the initial increase in TH expression and recovery of Ahd 2 and $N x p h 3$ expression could be caused by the upregulation of other bHLH factors present in the midbrain during development, we performed qPCR analyzes to examine the expression levels of Tcf3, Tcf12, Ngn2, and Ascl1 on dissected midbrain material at E14.5 (Figure 5A). Quantitative PCR on $T c f 4$ confirmed that $T c f 4$ was indeed lost in the Tcf4 mutant ( $\mathrm{n}=4 ; p=0.001$, two-tailed). Expression of Tcf3, Tcf12, and Ngn2 was not significantly altered in the midbrain of the Tcf4 mutant, indicating that if these genes compensated for Tcf4 loss of function, this was not controlled by upregulation of transcription of these factors. However, expression of Ascl1 was detected to be increased $\sim 1.8$-fold in the mutant midbrain at E14.5 ( $\mathrm{n}=4 ; p=0.05$, two-tailed). 
A

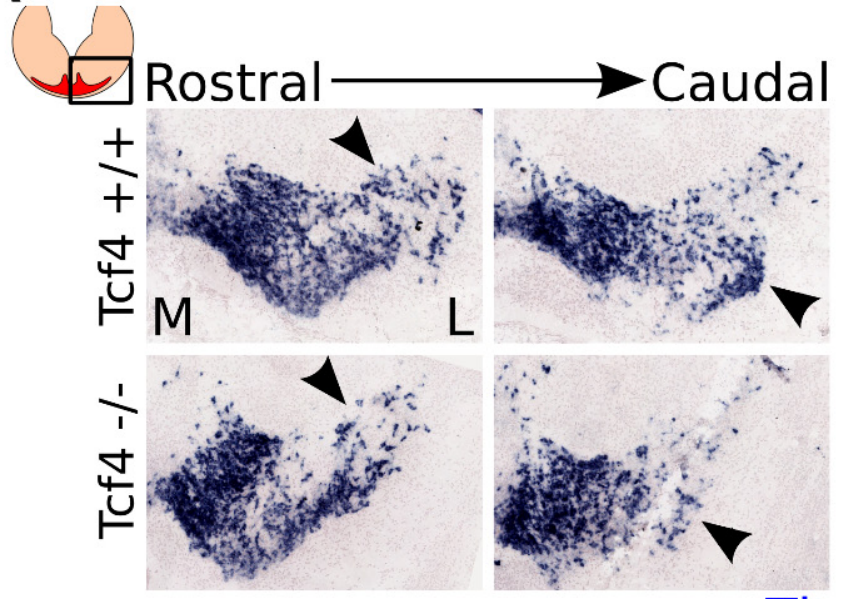

B

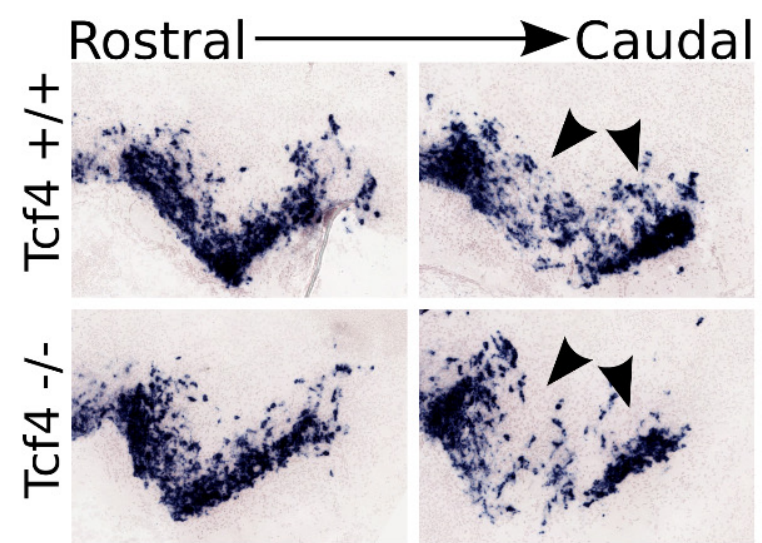

Th
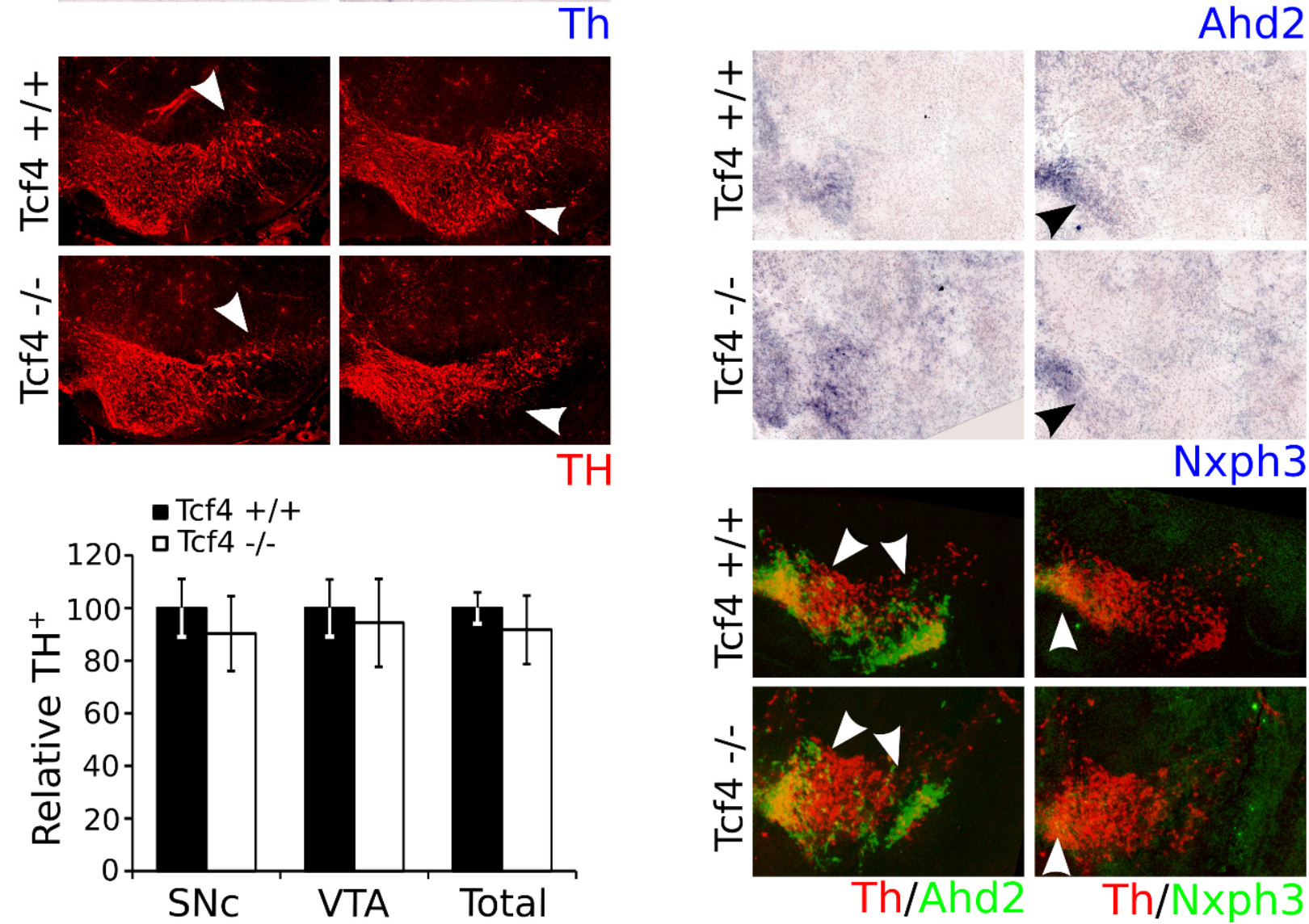



Th/Ahd2

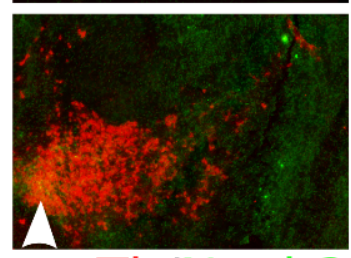

Th/Nxph3

Figure 4. Expression of $T h$ is affected in the mutant midbrain at P0, whereas the expression of Ahd2 and Nxph3 is partially recovered. (A) Expression of Th transcript (blue) in the mutant midbrain at P0 shows a decrease in the most lateral parts of the mdDA neuronal population (black arrowheads). Immunohistochemistry for TH-protein (red) shows a similar phenotype (white arrowheads). However, quantification of the TH-expressing neurons in the SNc, VTA, and the total population does not show a significant downregulation in the mutant (white bars) compared to the WT (black bars) ( $\mathrm{n}=3$, two-tailed) M: medial, L: lateral. (B) Ahd2 (blue) expression is mostly recovered, but still shows a decrease in expression in the caudal regions of the mdDA neuronal population (black arrowheads). Nxph3 (blue) expression is similarly mostly recovered, but still shows a decrease in expression in the caudal regions of the mdDA neuronal population (black arrowheads). Pseudooverlays of adjacent sections of Th (red) with Ahd2 (green) and Nxph3 (green) shows that the expression of Ahd2 and Nxph3 coincides with the expression of $T h$, and the affected areas are similar between the different markers (white arrowheads). 

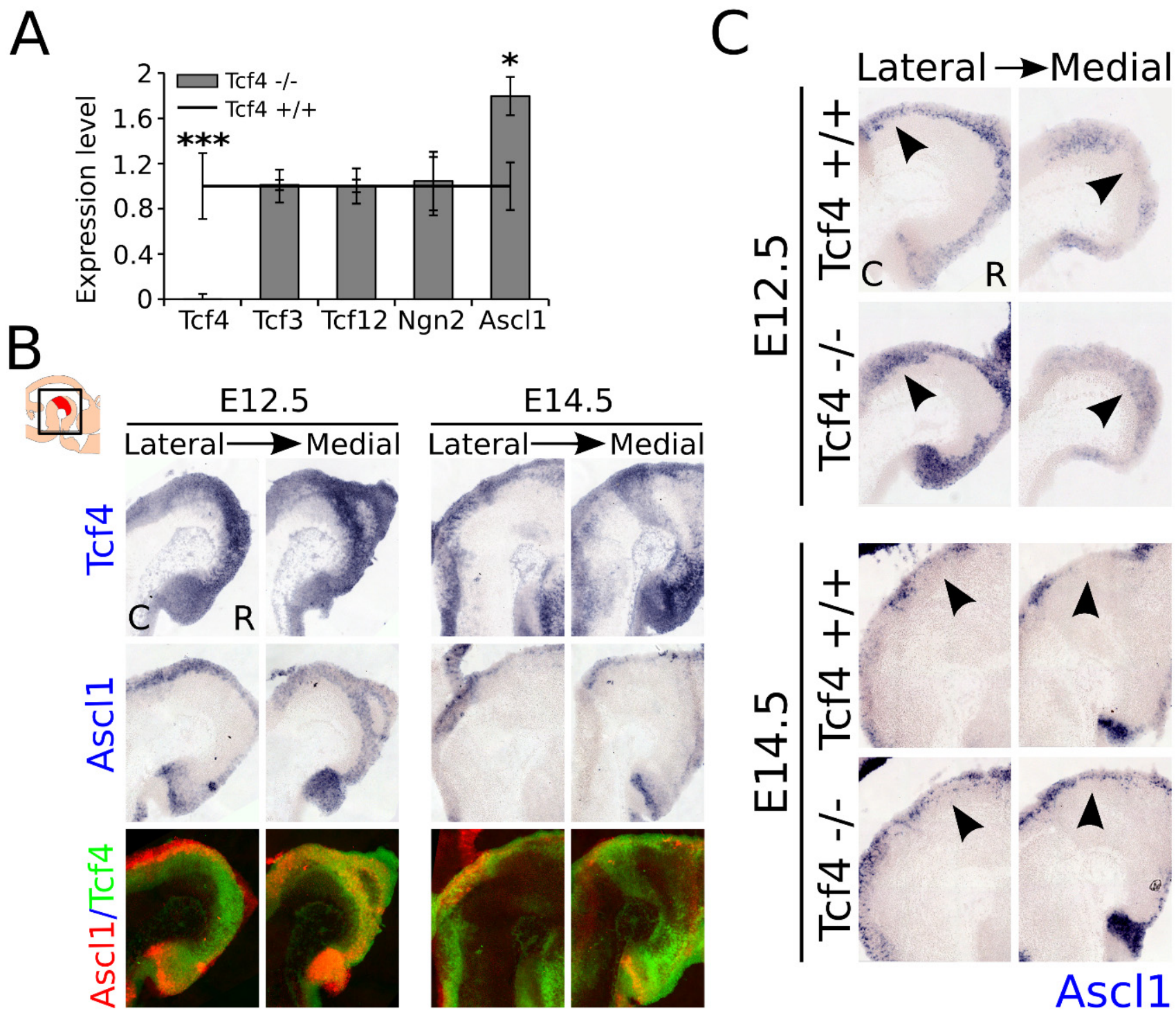

Figure 5. Ascl1 expression is upregulated in the Tcf4 mutant at E12.5 and E14.5. (A) The qPCR of E14.5 Tcf4 mutant and WT dissected midbrain material shows that in the mutant, the deleted part of the genome is indeed lost in the Tcf4 transcript (*** $\mathrm{n}=4 ; p=0.001$ two-tailed). Expression levels of different bHLH factors, Tcf3, Tcf12, Ngn2, and Ascl1, show that loss of Tcf4 leads to an upregulation of Ascl1 in the mutant midbrain at E14.5 ( ${ }^{*} \mathrm{n}=4 ; p=0.05$ two-tailed), whereas expression of the other bHLH factors in the midbrain remains unaffected. WT levels were set at 1. C: caudal, R: rostral. (B) WT expression of Tcf4 (blue) and Ascl1 (blue) shows an overlap in expression of areas at E12.5 and E14.5 in the VZ of the midbrain. However, at E14.5 the expression of Ascl1 appears to be absent in areas where Tcf4 is strongly expressed, which is clearly seen in the pseudo-overlays of the expression areas of Tcf4 (green) and Ascl1 (red) in adjacent sections. C: caudal, R: rostral. (C) In situ hybridization of Ascl1 (blue) on E12.5 and E14.5 embryonic midbrain shows an expanded area of expression of Ascl1 in the $\mathrm{VZ}$ of the embryonic midbrain in the mutant (black arrowheads). C: caudal, R: rostral.

To determine whether Tcf4 expression overlaps with the expression of Ascl1, we performed in situ hybridization on E12.5 and E14.5 WT material. Tcf4 expression strongly overlapped in the VZ of the midbrain at E12.5 with Ascl1 expression (Figure 5B). At E14.5, the expression of Tcf4 increased throughout the midbrain, whereas the expression of Ascl1 diminished at this stage. Pseudo-overlays of adjacent sections of Tcf4 and Ascl1 expression showed the overlap in expression at these developmental stages. Interestingly, at E14.5 the overlap with the expression of $A s c l 1$ seemed to decrease in the areas of the midbrain where Tcf4 was strongly expressed, suggesting a possible reciprocal expression at later stages. To examine whether Tcf4 normally inhibits the expression of Ascl1 or whether the 
loss of $T c f 4$ is compensated by an upregulation of Ascl1, we performed in situ hybridization for Ascl1 on E12.5 and E14.5 WT and mutant littermates (Figure 5C). Ascl1 showed an expanded expression pattern at both E12.5 and E14.5 in the VZ of the mutant midbrain (Figure 5C, black arrowheads). Together, these data indicated that the loss of Tcf4 resulted in an increase in expression of Ascl1, and that Tcf4 either regulated the expression of Ascl1, or that the loss of Tcf4 may have been partly compensated by an upregulation of this bHLH factor in the embryonic midbrain at the stages analyzed.

\section{Discussion}

Proneural bHLH factors like Ngn2, Ascl1, Tcf12, and Nato3 are known to be important in neuronal specification in the midbrain [14-17]. However, besides these bHLH factors, other members of the bHLH protein family of transcription factors are also expressed in the developing midbrain, like Tcf4, a member of the bHLH subfamily of E-box proteins. In this study, we focused on the role of Tcf4 in mdDA neuronal development. Tcf4 is expressed throughout the midbrain during development, but is no longer detected in the adult midbrain. During development, its expression is present in the VZ and overlaps with the rostral part of the TH-expressing population.

Loss of Tcf4 leads to an initial upward trend in the amount of TH-expressing cells early during development, which is recovered at E14.5, indicating that, different from what was detected in the rat [28], Tcf4 is not of major importance in the regulation of TH-expression in mdDA neurons in mice midbrain. However, as E-box binding sites are present in the promoter of the human, mouse, and rat Th gene, it may be of interest to determine whether TCF4 is able to bind to the promoter in the mouse brain and fine-tune Th expression in developing mdDA neurons. Interestingly, in a subset of embryos at E14.5, a change in expression of PITX3 protein was detected, which was not seen in earlier or later stages of development. The fact that this phenotype was only detected in some embryos and was not persistent throughout development suggests that the loss function of Tcf4 is not fully penetrating and may be influenced by genetic background and/or compensation by other bHLH factors.

Although the anatomical build-up of the mdDA neuronal domain seems relatively normal, we found that both Nxph3, a marker for the caudal mdDA subset, and Ahd2, a maker for the rostral mdDA subset, were changed in the Tcf4 mutant midbrain, indicating that the mdDA neuronal population is generated, but the identity of the different subsets is affected in the absence of Tcf4. These data suggest that Tcf4 has a specific role in subset specification. As the expression of both $N x p h 3$ and $A h d 2$ is mostly recovered at P0, loss of Tcf4 leads to a delayed subset specification. This regulation of subset identity is likely established in the VZ of the midbrain, or when mdDA neurons migrate through Tcf4 expressing areas toward their final location, as the expression of Tcf4 does not completely overlap with the expression areas of Nxph3 and Ahd2.

From earlier studies, it is known that bHLH factors can compensate for each other and regulate each other's expression in the embryonic midbrain [14,15]. Here, we detected that Ascl1 showed a significant upregulation during development as a consequence of Tcf4 ablation. Ascl1 is known to be important in the onset of mdDA neuronal differentiation [14,15]. The increase in expression of Ascl1 may possibly lead to a premature differentiation of neural progenitors into mdDA neurons, linking Tcf4 to the correct timing of mdDA neuronal differentiation. However, the delay in expression of the subset markers Ahd 2 and $N x p h 3$ suggests that the premature differentiation of TH-expressing neurons is only partial. Interestingly, although the expression of $A h d 2$ and Nxph3 is mostly recovered at P0, the expression of Th transcript and TH protein is visibly altered in lateral areas of the mdDA neuronal population, suggesting that the initial changed subset specification results in a permanent change in the mdDA neuronal population. As Tcf4 has been shown to affect axonal outgrowth and development of the major axonal tracts [27], it is possible that Tcf4 affects the projection of TH-expressing neurons to the striatum, eventually resulting in the degeneration of a subset of $\mathrm{TH}+$ neurons, as they cannot establish the correct connections. 
In the present study, we have established Tcf4 as a novel factor involved in the differentiation and specification of the mdDA neuronal population. Loss of Tcf4 may lead to premature neuronal differentiation, which has no apparent effect on the anatomical build-up and amount of TH-expressing cells at E14.5, although subset specification is initially affected. Interestingly, Tcf4 affects expression of Nxph3, a marker that was previously shown to be lowered in PD patients and an important factor in the survival of DA neurons derived from induced pluripotent stem cells, making Tcft an possible target in search of stem-cell-based therapies for PD [37].

Supplementary Materials: The following are available online at https://www.mdpi.com/2227-905 9/9/3/317/s1, Figure S1: Expression of TH is not visibly altered in the Tcf4 mutant at E12.5, Table S1: The qPCR sequences of primers used in this study.

Author Contributions: Conceptualization, S.M. and M.P.S.; methodology, S.M.; validation, S.M. and M.P.S.; formal analysis, S.M.; investigation, S.M. and I.W.; resources, S.M. and M.P.S.; data curation, S.M. and M.P.S.; writing—original draft preparation, S.M.; writing—review and editing, S.M. and M.P.S.; visualization, S.M.; supervision, M.P.S.; project administration, S.M. and M.P.S.; funding acquisition, M.P.S. All authors have read and agreed to the published version of the manuscript.

Funding: This research was funded by the Nederlandse Organisatie voor Wetenschappelijk Onderzoek-Aars en Levenswetenschappen (NOW-ALW) VICI grant (865.09.002) awarded to M.P.S.

Institutional Review Board Statement: The study was conducted according to the guidelines of the Declaration of Helsinki, and approved by the Institutional animal experimental committee (Dier ethische commissie) of the University of Amsterdam; DEC-UvA (DED237 (1 February 2012) and 2016-746-WP1 (20 January 2017)).

Data Availability Statement: The data presented in this study are available on request form the corresponding author(s).

Conflicts of Interest: The authors declare no conflict of interest. The funders had no role in the design of the study; in the collection, analyses, or interpretation of data; in the writing of the manuscript; or in the decision to publish the results.

\section{References}

1. Bayer, S.A.; Wills, K.V.; Triarhou, L.C.; Ghetti, B. Time of neuron origin and gradients of neurogenesis in midbrain dopaminergic neurons in the mouse. Exp. Brain Res. Exp. Hirnforsch. Exp. Céréb. 1995, 105, 191-199. [CrossRef]

2. Mesman, S.; von Oerthel, L.; Smidt, M.P. Mesodiencephalic dopaminergic neuronal differentiation does not involve GLI2Amediated SHH-signaling and is under the direct influence of canonical WNT signaling. PLoS ONE 2014, 9, e97926. [CrossRef]

3. Ono, Y.; Nakatani, T.; Sakamoto, Y.; Mizuhara, E.; Minaki, Y.; Kumai, M.; Hamaguchi, A.; Nishimura, M.; Inoue, Y.; Hayashi, H.; et al. Differences in neurogenic potential in floor plate cells along an anteroposterior location: Midbrain dopaminergic neurons originate from mesencephalic floor plate cells. Dev. Camb. Engl. 2007, 134, 3213-3225. [CrossRef]

4. Di Salvio, M.; Di Giovannantonio, L.G.; Acampora, D.; Prosperi, R.; Omodei, D.; Prakash, N.; Wurst, W.; Simeone, A. Otx2 controls neuron subtype identity in ventral tegmental area and antagonizes vulnerability to MPTP. Nat. Neurosci. 2010, 13, 1481-1488. [CrossRef]

5. Hoekstra, E.J.; Mesman, S.; de Munnik, W.A.; Smidt, M.P. LMX1B is part of a transcriptional complex with PSPC1 and PSF. PLoS ONE 2013, 8, e53122. [CrossRef] [PubMed]

6. Jacobs, F.M.J.; Veenvliet, J.V.; Almirza, W.H.; Hoekstra, E.J.; von Oerthel, L.; van der Linden, A.J.A.; Neijts, R.; Koerkamp, M.G.; van Leenen, D.; Holstege, F.C.P.; et al. Retinoic acid-dependent and -independent gene-regulatory pathways of Pitx3 in meso-diencephalic dopaminergic neurons. Dev. Camb. Engl. 2011, 138, 5213-5222. [CrossRef] [PubMed]

7. Smits, S.M.; von Oerthel, L.; Hoekstra, E.J.; Burbach, J.P.H.; Smidt, M.P. Molecular Marker Differences Relate to Developmental Position and Subsets of Mesodiencephalic Dopaminergic Neurons. PLoS ONE 2013, 8, e76037. [CrossRef] [PubMed]

8. Veenvliet, J.V.; Dos Santos, M.T.M.A.; Kouwenhoven, W.M.; von Oerthel, L.; Lim, J.L.; van der Linden, A.J.A.; Koerkamp, M.J.A.G.; Holstege, F.C.P.; Smidt, M.P. Specification of dopaminergic subsets involves interplay of En1 and Pitx3. Dev. Camb. Engl. 2013, 140, 3373-3384. [CrossRef]

9. Barzilai, A.; Melamed, E. Molecular mechanisms of selective dopaminergic neuronal death in Parkinson's disease. Trends Mol. Med. 2003, 9, 126-132. [CrossRef]

10. Mesman, S.; Smidt, M.P. Acquisition of the Midbrain Dopaminergic Neuronal Identity. Int. J. Mol. Sci. 2020, 21, 4638. [CrossRef]

11. Skinner, M.K.; Rawls, A.; Wilson-Rawls, J.; Roalson, E.H. Basic helix-loop-helix transcription factor gene family phylogenetics and nomenclature. Differ. Res. Biol. Divers. 2010, 80, 1-8. [CrossRef] 
12. Murre, C.; McCaw, P.S.; Vaessin, H.; Caudy, M.; Jan, L.Y.; Jan, Y.N.; Cabrera, C.V.; Buskin, J.N.; Hauschka, S.D.; Lassar, A.B. Interactions between heterologous helix-loop-helix proteins generate complexes that bind specifically to a common DNA sequence. Cell 1989, 58, 537-544. [CrossRef]

13. Powell, L.M.; Jarman, A.P. Context dependence of proneural bHLH proteins. Curr. Opin. Genet. Dev. 2008, 18, 411-417. [CrossRef]

14. Andersson, E.; Jensen, J.B.; Parmar, M.; Guillemot, F.; Björklund, A. Development of the mesencephalic dopaminergic neuron system is compromised in the absence of neurogenin 2. Dev. Camb. Engl. 2006, 133, 507-516. [CrossRef] [PubMed]

15. Kele, J.; Simplicio, N.; Ferri, A.L.M.; Mira, H.; Guillemot, F.; Arenas, E.; Ang, S.-L. Neurogenin 2 is required for the development of ventral midbrain dopaminergic neurons. Dev. Camb. Engl. 2006, 133, 495-505. [CrossRef] [PubMed]

16. Mesman, S.; Smidt, M.P. Tcf12 Is Involved in Early Cell-Fate Determination and Subset Specification of Midbrain Dopamine Neurons. Front. Mol. Neurosci. 2017, 10, 353. [CrossRef]

17. Peterson, D.J.; Marckini, D.N.; Straight, J.L.; King, E.M.; Johnson, W.; Sarah, S.S.; Chowdhary, P.K.; DeLano-Taylor, M.K. The Basic Helix-Loop-Helix Gene Nato3 Drives Expression of Dopaminergic Neuron Transcription Factors in Neural Progenitors. Neuroscience 2019, 421, 176-191. [CrossRef]

18. Massari, M.E.; Murre, C. Helix-loop-helix proteins: Regulators of transcription in eucaryotic organisms. Mol. Cell. Biol. 2000, 20, 429-440. [CrossRef]

19. Wojciechowski, J.; Lai, A.; Kondo, M.; Zhuang, Y. E2A and HEB are required to block thymocyte proliferation prior to pre-TCR expression. J. Immunol. Baltim. Md 1950 2007, 178, 5717-5726. [CrossRef]

20. Wikström, I.; Forssell, J.; Penha-Goncalves, M.N.; Bergqvist, I.; Holmberg, D. A role for E2-2 at the DN3 stage of early thymopoiesis. Mol. Immunol. 2008, 45, 3302-3311. [CrossRef]

21. Fischer, B.; Azim, K.; Hurtado-Chong, A.; Ramelli, S.; Fernández, M.; Raineteau, O. E-proteins orchestrate the progression of neural stem cell differentiation in the postnatal forebrain. Neural Develop. 2014, 9, 23. [CrossRef] [PubMed]

22. Ravanpay, A.C.; Olson, J.M. E protein dosage influences brain development more than family member identity. J. Neurosci. Res. 2008, 86, 1472-1481. [CrossRef] [PubMed]

23. Sweatt, J.D. Pitt-Hopkins Syndrome: Intellectual disability due to loss of TCF4-regulated gene transcription. Exp. Mol. Med. 2013, 45, e21. [CrossRef] [PubMed]

24. Zollino, M.; Zweier, C.; Van Balkom, I.D.; Sweetser, D.A.; Alaimo, J.; Bijlsma, E.K.; Cody, J.; Elsea, S.H.; Giurgea, I.; Macchiaiolo, M.; et al. Diagnosis and management in Pitt-Hopkins syndrome: First international consensus statement. Clin. Genet. 2019, 95, 462-478. [CrossRef]

25. Flora, A.; Garcia, J.J.; Thaller, C.; Zoghbi, H.Y. The E-protein Tcf4 interacts with Math1 to regulate differentiation of a specific subset of neuronal progenitors. Proc. Natl. Acad. Sci. USA 2007, 104, 15382-15387. [CrossRef]

26. Li, H.; Zhu, Y.; Morozov, Y.M.; Chen, X.; Page, S.C.; Rannals, M.D.; Maher, B.J.; Rakic, P. Disruption of TCF4 regulatory networks leads to abnormal cortical development and mental disabilities. Mol. Psychiatry 2019, 24, 1235-1246. [CrossRef]

27. Mesman, S.; Bakker, R.; Smidt, M.P. Tcf4 is required for correct brain development during embryogenesis. Mol. Cell. Neurosci. 2020, 106, 103502. [CrossRef]

28. Yoon, S.O.; Chikaraishi, D.M. Isolation of two E-box binding factors that interact with the rat tyrosine hydroxylase enhancer. J. Biol. Chem. 1994, 269, 18453-18462. [CrossRef]

29. Smidt, M.P.; Smits, S.M.; Bouwmeester, H.; Hamers, F.P.T.; van der Linden, A.J.A.; Hellemons, A.J.C.G.M.; Graw, J.; Burbach, J.P.H. Early developmental failure of substantia nigra dopamine neurons in mice lacking the homeodomain gene Pitx3. Dev. Camb. Engl. 2004, 131, 1145-1155. [CrossRef]

30. Fenstermaker, A.G.; Prasad, A.A.; Bechara, A.; Adolfs, Y.; Tissir, F.; Goffinet, A.; Zou, Y.; Pasterkamp, R.J. Wnt/planar cell polarity signaling controls the anterior-posterior organization of monoaminergic axons in the brainstem. J. Neurosci. Off. J. Soc. Neurosci. 2010, 30, 16053-16064. [CrossRef]

31. Kolk, S.M.; Gunput, R.-A.F.; Tran, T.S.; van den Heuvel, D.M.A.; Prasad, A.A.; Hellemons, A.J.C.G.M.; Adolfs, Y.; Ginty, D.D.; Kolodkin, A.L.; Burbach, J.P.H.; et al. Semaphorin 3F is a bifunctional guidance cue for dopaminergic axons and controls their fasciculation, channeling, rostral growth, and intracortical targeting. J. Neurosci. Off. J. Soc. Neurosci. 2009, 29, 12542-12557. [CrossRef] [PubMed]

32. Smidt, M.P.; Asbreuk, C.H.J.; Cox, J.J.; Chen, H.; Johnson, R.L.; Burbach, J.P.H. A second independent pathway for development of mesencephalic dopaminergic neurons requires Lmx1b. Nat. Neurosci. 2000, 3, 337-341. [CrossRef]

33. Bye, C.R.; Thompson, L.H.; Parish, C.L. Birth dating of midbrain dopamine neurons identifies A9 enriched tissue for transplantation into Parkinsonian mice. Exp. Neurol. 2012, 236, 58-68. [CrossRef]

34. Zhuang, Y.; Cheng, P.; Weintraub, H. B-lymphocyte development is regulated by the combined dosage of three basic helix-loophelix genes, E2A, E2-2, and HEB. Mol. Cell. Biol. 1996, 16, 2898-2905. [CrossRef] [PubMed]

35. Tiklová, K.; Björklund, Å.K.; Lahti, L.; Fiorenzano, A.; Nolbrant, S.; Gillberg, L.; Volakakis, N.; Yokota, C.; Hilscher, M.M.; Hauling, T.; et al. Single-Cell RNA Sequencing Reveals Midbrain Dopamine Neuron Diversity Emerging during Mouse Brain Development. Nat. Commun. 2019, 10, 581. [CrossRef] [PubMed] 
36. Hoekstra, E.J.; von Oerthel, L.; van der Heide, L.P.; Kouwenhoven, W.M.; Veenvliet, J.V.; Wever, I.; Jin, Y.-R.; Yoon, J.K.; van der Linden, A.J.A.; Holstege, F.C.P.; et al. Lmx1a Encodes a Rostral Set of Mesodiencephalic Dopaminergic Neurons Marked by the Wnt/B-Catenin Signaling Activator R-spondin 2. PLoS ONE 2013, 8, e74049. [CrossRef]

37. Nishimura, K.; Murayama, S.; Takahashi, J. Identification of Neurexophilin 3 as a Novel Supportive Factor for Survival of Induced Pluripotent Stem Cell-Derived Dopaminergic Progenitors. Stem Cells Transl. Med. 2015, 4, 932-944. [CrossRef] [PubMed] 